\title{
Distribution Problems, Their Modifications and Applications
}

\author{
MILOŠ ŠEDA, JAN ROUPEC \\ Institute of Automation and Computer Science \\ Brno University of Technology \\ Technická 2, 61669 Brno, Czech Republic \\ seda@fme.vutbr.cz,roupec@fme.vutbr.cz
}

\author{
JINDŘIŠKA ŠEDOVÁ \\ Faculty of Economics and Administration \\ Masaryk University \\ Lipová 41a, 60200 Brno, Czech Republic \\ jsedova@econ.muni.cz
}

\begin{abstract}
In this paper, we deal with well-known distribution problems and discuss their restrictions, extensions and modifications including a possible application in agriculture. We show that the transportation problem can be transformed to an assignment problem using special constraints, but because of NPhardness it needs quite different methods of its solving. Another modification of the transportation problem, the crop problem, has an application in agriculture, but we must deal with uncertain data. We propose a genetic algorithm and fuzzy logic approach for solving these problems.
\end{abstract}

Keywords - transportation; assignment problem; crop problem, PERT; heuristic; genetic algorithm; fuzzy number

\section{INTRODUCTION}

The Hitchcock transportation problem with $m$ sources (supply points, factories) and $n$ destinations (demand points, clients) can be formulated using linear programming as follows [1]:

Minimise

$$
z=\sum_{i=1}^{m} \sum_{j=1}^{n} c_{i j} x_{i j}
$$

subject to

$$
\begin{gathered}
\sum_{j=1}^{n} x_{i j}=a_{i}, \quad i=1, \ldots, m \\
\sum_{i=1}^{m} x_{i j}=b_{j}, \quad j=1, \ldots, n \\
x_{i j} \geq 0, \quad i=1, \ldots, m, j=1, \ldots, n,
\end{gathered}
$$

where

$z$ is the total transportation cost,

$c_{i j}$ is the unit shipping cost from source $i$ to destination $j$,

$x_{i j}$ is the number of units shipped from source $i$ to destination $j$,

$a_{i}$ is the supply of the source $i$,

$b_{j}$ is the demand of destination $j$, and only a single commodity is transported.

Since there is only one commodity, a d estination can receive its demand from more than one source. Therefore, the objective is to determine how much should be shipped from each source to each destination so as to minimise the total transportation cost.

If total commodity supply equals to total demand, the problem is said to be a balanced transportation problem:

$$
\sum_{i=1}^{m} a_{i}=\sum_{j=1}^{n} b_{j}
$$

If (5) is not satisfied, then it becomes an unbalanced transportation problem.

$$
\sum_{i=1}^{m} a_{i}>\sum_{j=1}^{n} b_{j}
$$

If total supply exceeds total demand, see (6), we can balance the problem by adding a dummy destination to absorb the excess supply. Shipments to this destination are assigned a cost of zero.

$$
\sum_{i=1}^{m} a_{i}<\sum_{j=1}^{n} b_{j}
$$

If a transportation problem has a total supply that is strictly less than total demand, see (7), the problem has no feasible solution, because one or more demands cannot be satisfied. In such situations a penalty cost is often associated with unmet demand and the total penalty cost is desired to be minimal.

There are several methods for solving the balanced transportation problem as follows:

- The Northwest Corner Method

- The Least Cost Method

- Vogel's Approximation Method

We can also solve the transportation problem using specialised software tools, e.g. GAMS, LINDO, LINGO, or MS Excel Solver. 
If it is possible to both ship into and out of the same point of the transport network, then we speak about a transshipment (or transhipment) problem. For the transshipment problem, you can ship from one supply point to another or from one demand point to another.

The Hitchcock formulation of the transportation problem may also be extended considering fixed charges associated with supply points (e. g. warehouses), means of transport, their capacity, cost of transport by vehicles to $1 \mathrm{~km}$, which enables to determine the number of trips due to volume, transport in two levels: primary source - warehouses - destinations, admitting the possibility of direct transport from the primary source to destinations, etc.

Instead, we turn our attention to problems that seem to have nothing with transportation, but their formulation can be obtained from the basic model of the transportation problem.

\section{ASSIGNMENT PROBLEM}

Let us assume a balanced transportation problem in which all supplies and demands are equal to 1 and the same number of sources and destinations [2].

An example of this situation is an assignment of tasks to persons or jobs to machines. Additionally, we assume that each person must do on e and only one task, and each task must be done by only one person. The problem is to find an assignment which minimizes the total cost (or the total time) for processing all tasks.

\section{Consider the following denotations:}

$n=$ number of persons $=$ number of tasks,

$x_{i j}$ represents the assignment of person $i$ to task $j, x_{i j}=1$ if the assignment is done, and $x_{i j}=0$ otherwise,

$c_{i j}$ is the cost the assignment of person $i$ to task $j$.

Hence we get the following model of the (linear) assignment problem (AP):

Minimise

$$
z=\sum_{i=1}^{m} \sum_{j=1}^{n} c_{i j} x_{i j}
$$

subject to

$$
\begin{gathered}
\sum_{j=1}^{n} x_{i j}=1, \quad i=1, \ldots, n \\
\sum_{i=1}^{n} x_{i j}=1, \quad j=1, \ldots, n \\
x_{i j} \in\{0,1\}, \quad i=1, \ldots, n, j=1, \ldots, n
\end{gathered}
$$

We can see that:

(1) The assignment problem is a special case of the transportation problem where

$$
\begin{aligned}
& m=n, \\
& a_{i}=1, i=1,2, \ldots, m, \\
& b_{j}=1, j=1,2, \ldots, n, \\
& x_{i j} \in\{0,1\}, i=1,2, \ldots, m, j=1,2, \ldots, n .
\end{aligned}
$$

(2) The assignment problem can also be understood as a problem of finding a permutation

$$
\left(\begin{array}{cccc}
1 & 2 & \ldots & n \\
\pi_{1} & \pi_{2} & \ldots & \pi_{n}
\end{array}\right)
$$

where person $i$ to task $\pi_{i}$ is assigned, $i=1, \ldots, n$, and

$$
\sum_{i=1}^{n} c_{i, \pi_{i}} \rightarrow \min
$$

Since there are $n$ ! permutations for $n$ tasks, finding the solution for large instances is not reachable in a reasonable amount of time, and thus heuristic methods must be used. We present a genetic algorithm approach.

\section{GENETIC ALGORITHM FOR AP}

The skeleton for GA can be described as follows [3]:

generate an initial population ;

evaluate fitness of individuals in the population ;

repeat

select parents from the population;

recombine (mate) parents to produce children ;

evaluate fitness of the children ;

replace some or all of the population by the children until a satisfactory solution has been found ;

Since the principles of GAs are well-known, we will only deal with GA parameter settings for the problems to be studied. Now we describe the general settings [4], [5].

Individuals in the population (chromosomes) are represented as binary strings of length $n$, where a value of 0 or 1 at the $i$-th bit (gene) implies that $x_{i}=0$ or 1 in the solution respectively.

The population size $N$ is usually set between $n$ and $2 n$. Many empirical results have shown that population sizes in the range $[50,200]$ work quite well for most problems.

Initial population is obtained by generating random strings of $0 \mathrm{~s}$ and $1 \mathrm{~s}$ in the following way: First, all bits in all strings are set to 0 , and then, for each of the strings, randomly selected bits are set to 1 until the solutions (represented by strings) are feasible.

The fitness function corresponds to the objective function to be maximised or minimised.

There are three most commonly used methods of selection of two parent solution for reproduction: proportionate selection, ranking selection, and tournament selection. The tournament selection is perhaps the simplest and most efficient among these three methods. We use the binary tournament selection method where two individuals are chosen randomly from the population. The more fit individual is then allocated a 
reproductive trial. In order to produce a child, two binary tournaments are held, each of which produces one parent.

The recombination is provided by the uniform crossover operator, which has a better recombination potential than do other crossover operators as the classical one-point and twopoint crossover operators. The uniform crossover operator works by generating a random crossover mask $B$ (using Bernoulli distribution) which can be represented as a binary string $B=b_{1} b_{2} b_{3} \cdots b_{n-1} b_{n}$ where $n$ is the length of the chromosome. Let $P_{1}$ and $P_{2}$ be the parent strings $P_{1}[1], \ldots$ ,$P_{1}[n]$ and $P_{2}[1], \ldots, P_{2}[n]$ respectively. Then the child solution is created by letting: $C[i]=P_{1}[i]$ if $b_{i}=0$ and $C[i]=P_{2}[i]$ if $b_{i}$ $=1$. Mutation is applied to each child after crossover. It works by inverting $M$ randomly chosen bits in a string where $M$ is experimentally determined. We use a mutation rate of $5 / n$ as a lower bound on the optimal mutation rate. It is equivalent to mutating five randomly chosen bits per string.

When $v$ child solutions have been generated, the children will replace $v$ members of the existing population to keep the population size constant, and the reproductive cycle will restart. As the replacement of the whole parent population does not guarantee that the best member of a population will survive into the next generation, it is better to use steady-state or incremental replacement which generates and replaces only a few members (typically 1 or 2 ) of the population during each generation. The least-fit member, or a randomly selected member with below-average fitness, are usually chosen for replacement.

Termination of a $G A$ is usually controlled by specifying a maximum number of generations $t_{\max }$ or relative improvement of the best objective function value over generations. Since the optimal solution values for most problems are not known, we choose $t_{\max } \leq 5000$.

In our implementation the population was set to 50 and the number of iterations to $10 \times n^{2}$.

Permutations of $n$ tasks for the initial population are best to be generated randomly, e.g. by the following procedure:

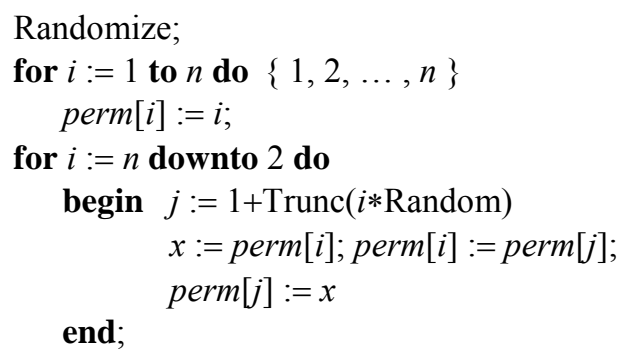

As to the crossover operation, we cannot use the traditional two-point crossover, because it would lead to infeasible solutions. If we change the middle parts of the parent chromosomes $\quad P_{1}=(1,10,7,2,8,9,4,6,5,3)$, $P_{2}=(5,8,2,9,7,4,1,10,3,6)$ between the 4-th and 7-th position, then we would obtain offspring $(1,10,7,9,7,4,1,6,5,3)$ and $(5,8,2,2,8,9,4,10,3,6)$ that correspond to no permutations, because some jobs are duplicated or omitted. We used the so called crossover in a $\mathrm{p}$ artially mapped representation where the genes in the middle part of one chromosome are ordered in its offspring by their occurrence in the second parent chromosome.

In the literature, slight modifications of these shift operations can be found, e.g. 1stSwap, FullSwap, DoubleCut, DoublePointShift and RightDoublePoint [6], [7].

Genetic algorithms may be enhanced by other heuristic methods to perform local searches. A sophisticated approach was presented in [8].

First, define the distance of two permutations $P_{1}, P_{2}$. The most frequent definitions are the following

- $\quad$ precedence distance - computed as the number of pairs of jobs $\{i, j\}$ where $i$ precedes $j$ in $P_{1}$, but does not precede it in $P_{2}$,

- $\quad$ positional distance - given as the sum of differences of jobs on the same positions in both permutations.

This method combines the genetic algorithm with a local search and the Metropolis criterion used in simulated annealing. Its main modification is based on a secial crossover, called multistep crossover fusion (MSXF). Let $P_{1}$ and $P_{2}$ be two parent permutations. On permutation $P_{1}$ a local search is applied that is, to a cer tain extent, influenced by permutation $P_{2}$, which serves a r eference point. All permutations from a neighbourhood of $P_{1}$ are ordered in the ascending order by their distance from permutation $P_{2}$. With a probability inversely proportional to this sequence, a neighbour, $P_{s}$, is selected. If this neighbour solution gives a shorter schedule than the one computed from the permutation of jobs $P_{1}$, i.e. $C_{\max }\left(P_{s}\right)<C_{\max }\left(P_{1}\right)$, then $P_{s}$ is accepted. In the opposite case, it is accepted with the probability of $e^{-\left(C_{\max }\left(P_{\mathrm{s}}\right)-C_{\max }\left(P_{1}\right)\right) / T}$ as in the Metropolis criterion. For simplicity, the temperature $T$ is considered constant. The selection of neighbours is repeated until a neighbour is selected. The number of iterations is also set fixed. From all the accepted permutations, only the best one is included in the next iteration of the genetic algorithm.

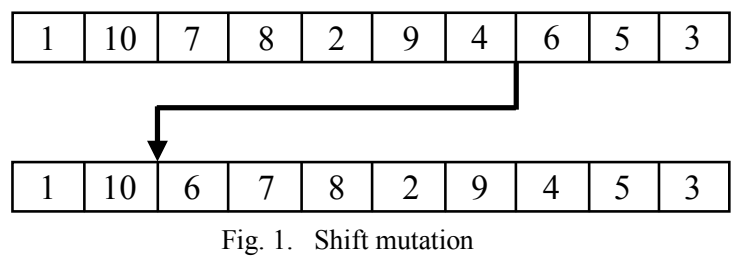

For mutation we considered three operators:

- exchange mutation (it exchanges two randomly selected positions in a permutation),

- $\quad$ shift mutation (it removes a value at one position and puts it at another position), see Fig. 1, and

- mutation inspired by well-known Lin-2-Opt change operator usually used for solving the travelling salesman problem [9]. Here first two elements are added to the permutation (into positions 0 and $|n|+1$ ) and then the same values are assigned to them to 
simulate a cyclic tour. Two 'edges' (pairs of neighbour elements in permutation) are randomly chosen $\left(\left(p_{1}, p_{2}\right)\right.$ and $\left(q_{1}, q_{2}\right)$ say $)$, the inner elements $p_{2}, q_{1}$ are swapped and the elements between $p_{2}$ and $q_{1}$ are reversed.

The best results were achieved with the shift mutation.

\section{CROP PROBLEM}

Let us denote:

$p_{1}, \ldots, p_{m}=$ grounds

$r_{1}, \ldots, r_{m}=$ area of grounds

$k_{1}, \ldots, k_{n}=$ crops

$c_{i j}, i=1,2, \ldots, m, j=1,2, \ldots, n$

$=$ profit from 1 ha of ground sown by crop $k_{j}$

$x_{i j}=$ number of hectares of ground $p_{i}$ sown by crop $k_{j}$

TABLE I. CROP PROBLEM

\begin{tabular}{|l|l|l|l|l|l|}
\hline $\begin{array}{c}\text { crops } \\
\text { grounds }\end{array}$ & $\boldsymbol{k}_{\mathbf{1}}$ & $\boldsymbol{k}_{\mathbf{2}}$ & & $\boldsymbol{k}_{\boldsymbol{n}}$ & area [ha] \\
\hline $\boldsymbol{p}_{\mathbf{1}}$ & $c_{11}$ & $c_{12}$ & $\cdots$ & $c_{1 n}$ & $r_{1}$ \\
\hline $\boldsymbol{p}_{2}$ & $c_{21}$ & $c_{22}$ & $\cdots$ & $c_{2 n}$ & $r_{2}$ \\
\hline$\cdots$ & & & & & $\cdots$ \\
\hline $\boldsymbol{p}_{\boldsymbol{m}}$ & $c_{m 1}$ & $c_{m 2}$ & $\cdots$ & $c_{\mathrm{m} n}$ & $r_{m}$ \\
\hline
\end{tabular}

In the crop problem [10], [11] is to find the optimum sowing of areas by crops for given yields of crops (in quintals per hectare) and contractual purchase prices so as to maximise the total profit [12].

From Table I we get the following system of equations:

$$
\begin{array}{ccc}
x_{11}+x_{12}+\ldots+x_{1 n} & \leq & r_{1} \\
x_{21}+x_{22}+\ldots+x_{2 n} & \leq & r_{2} \\
\ldots & & \\
x_{m 1}+x_{m 2}+\ldots+x_{m n} & \leq & r_{m} \\
x_{i j} \geq 0, \quad i=1,2, \ldots, m, j=1,2, \ldots, n & \\
z=c_{11} x_{11}+c_{12} x_{12}+\ldots+c_{1 n} x_{1 n}+c_{21} x_{21}+c_{22} x_{22}+\ldots+ \\
c_{2 n} x_{2 n}+\ldots+c_{m 1} x_{m 1}+c_{m 2} x_{m 2}+\ldots+c_{m n} x_{m n} \rightarrow \max
\end{array}
$$

It can be expressed as follows:

Maximise

$$
z=\sum_{i=1}^{m} \sum_{j=1}^{n} c_{i j} x_{i j}
$$

subject to

$$
\begin{gathered}
\sum_{j=1}^{n} x_{i j} \leq r_{i}, \quad i=1, \ldots, m \\
x_{i j} \geq 0, \quad i=1, \ldots, m, j=1, \ldots, n
\end{gathered}
$$

If we require for each crop that were sown at a cer tain minimum area, then the task has become an example of maximisation version of the generalized distribution problem. It is included in Table II and the corresponding model follows:

TABLE II. CRop Problem With Minimum ReQUiREMENTS

\begin{tabular}{|l|l|l|l|l|l|}
\hline $\begin{array}{l}\text { crops } \\
\text { grounds }\end{array}$ & $\boldsymbol{k}_{\mathbf{1}}$ & $\boldsymbol{k}_{\mathbf{2}}$ & & $\boldsymbol{k}_{\boldsymbol{n}}$ & area [ha] \\
\hline $\boldsymbol{p}_{\mathbf{1}}$ & $c_{11}$ & $c_{12}$ & $\cdots$ & $c_{1 n}$ & $r_{1}$ \\
\hline $\boldsymbol{p}_{\mathbf{2}}$ & $c_{21}$ & $c_{22}$ & $\cdots$ & $c_{2 n}$ & $r_{2}$ \\
\hline$\cdots$ & & & & & $\cdots$ \\
\hline $\boldsymbol{p}_{\boldsymbol{m}}$ & $c_{m 1}$ & $c_{m 2}$ & $\cdots$ & $c_{\mathrm{m} n}$ & $r_{m}$ \\
\hline $\begin{array}{l}\text { minimum } \\
\text { requirements for } \\
\text { crop sowing area }\end{array}$ & $\boldsymbol{d}_{\mathbf{1}}$ & $\boldsymbol{d}_{\mathbf{2}}$ & & $\boldsymbol{d}_{\boldsymbol{n}}$ & \\
\hline
\end{tabular}

Maximise

$$
z=\sum_{i=1}^{m} \sum_{j=1}^{n} c_{i j} x_{i j}
$$

subject to

$$
\begin{gathered}
\sum_{j=1}^{n} x_{i j} \leq r_{i}, \quad i=1, \ldots, m \\
\sum_{i=1}^{m} x_{i j} \geq d_{j}, \quad j=1, \ldots, n \\
x_{i j} \geq 0, \quad i=1, \ldots, m, j=1, \ldots, n
\end{gathered}
$$

\section{CROP PROBLEM WITH UNCERTAIN YIELDS}

Of course, yields of crops are only estimated and in real conditions cannot be considered as deterministic.

This situation can be solved with techniques inspired by PERT.

\section{Denote}

$a=$ estimate of the crop yields under the most favourable conditions

$b=$ estimate of the crop yields under the least favourable conditions

$m=$ most likely value for the crop yields 
PERT requires the assumption that estimated parameter follows a beta distribution. Then its mean values may be approximated by the following equation:

$$
y=\frac{a+4 m+b}{6}
$$

Since the beta distribution is not guaranteed, we propose a fuzzy approach.

Let us assume now that crop yields are given by fuzzy numbers [13].

A fuzzy number $A$ is a fuzzy set represented by 4-tuple $\left(a_{1}, a_{2}, a_{3}, a_{4}\right)$ and a piecewise continuous membership function with the following properties:

- $a_{1} \leq a_{2} \leq a_{3} \leq a_{4}$

- $\mu_{A(x)}=0$ for $x \leq a_{1}, x \geq a_{4}$

- $\mu_{A(x)}=1$ for $a_{2} \leq x \leq a_{3}$

- $\mu_{A}$ is increasing on $\left[a_{1}, a_{2}\right]$ and decreasing on $\left[a_{3}, a_{4}\right]$.

The fuzzy set defined by the membership function is an example of fuzzy number. In this paragraph we consider trapezoidal fuzzy numbers, see (22) and Fig. 2.

$$
\mu_{A}(x)
$$

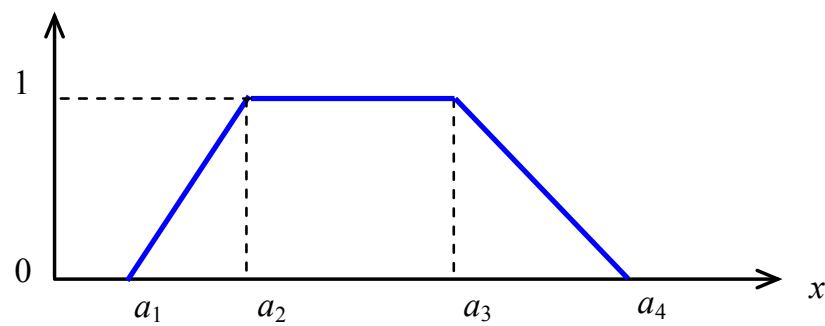

$\Pi(x, a, b, c, d)=\left\{\begin{array}{lc}0 & \text { for } x<a_{1} \\ \frac{x-a_{1}}{a_{2}-a_{1}} & \text { for } a_{1} \leq x \leq a_{2} \\ 1 & \text { for } a_{2}<x \leq a_{3} \\ \frac{a_{3}-x}{a_{4}-a_{3}} & \text { for } a_{3}<x \leq a_{4} \\ 0 & \text { for } x>a_{4}\end{array}\right.$

Fig. 2. Trapezoidal fuzzy number
The addition of fuzzy numbers can be derived using the extension principle and it is determined as follows:

$$
\begin{array}{r}
X^{F} \oplus Y^{F}=\left(x_{1}, x_{2}, x_{3}, x_{4}\right) \oplus\left(y_{1}, y_{2}, y_{3}, y_{4}\right)= \\
=\left(x_{1}+y_{1}, x_{2}+y_{2}, x_{3}+y_{3}, x_{4}+y_{4}\right)
\end{array}
$$

When the maximum operation would be derived in the same way, then its results may not be trapezoidal fuzzy numbers. Therefore we approximate this operation as follows.

$\max \left(X^{F}, Y^{F}\right)=\left(\max \left(x_{1}, y_{1}\right), \max \left(x_{2}, y_{2}\right), \max \left(x_{3}, y_{3}\right), \max \left(x_{4}, y_{4}\right)\right)$ (3.20)

To find a solution of the crop problem which maximises the total profit, we must compare fuzzy numbers in some way, which is a difficult problem. An ordering relation $\leq$ can be defined e.g. as follows:

$$
X^{F} \leq Y^{F} \Leftrightarrow\left(x_{1} \leq y_{1}\right) \wedge\left(x_{2} \leq y_{2}\right) \wedge\left(x_{3} \leq y_{3}\right) \wedge\left(x_{4} \leq y_{4}\right)
$$

However, this relation is not a complete ordering relation, as fuzzy numbers $X^{F}, Y^{F}$ satisfying

$$
(\exists i, j \in\{1,2,3,4\}):\left(x_{i}<y_{i}\right) \wedge\left(x_{j}>y_{j}\right)
$$

are not comparable by $\leq$.

It is evident that, for non-comparable fuzzy numbers $X^{F}$, $Y^{F}$, this fuzzy max operation results in a fuzzy number different from both of them. For example, for $X^{F}=(4,9,12,16)$ and $Y^{F}=(6,8,13,15)$, we get from $(23)$ a fuzzy $\max (6,9,13,16)$ which differs from $X^{F}$ and $Y^{F}$.

This problem can be solved by assigning a scalar value to each resulting fuzzy number and comparing these scalars.

We use the fuzzy ranking method described in [14], modified for the case of trapezoidal fuzzy numbers. This method uses inverse functions

$g_{A}^{L}:[0,1] \rightarrow\left[a_{1}, a_{2}\right]$ and $g_{A}^{R}:[0,1] \rightarrow\left[a_{3}, a_{4}\right]$ derived from functions $f_{A}^{L}:\left[a_{1}, a_{2}\right] \rightarrow[0,1] \quad$ and $\quad f_{A}^{R}:\left[a_{3}, a_{4}\right] \rightarrow[0,1]$, respectively.

From $y=\frac{x-a_{1}}{a_{2}-a_{1}}$ (increasing part) and $y=\frac{x-a_{4}}{a_{3}-a_{4}}$ (decreasing part) we can derive that

$$
g_{A}^{L}=a_{1}+\left(a_{2}-a_{1}\right) y, \quad g_{A}^{R}=a_{4}+\left(a_{3}-a_{4}\right) y
$$

The ranking function is defined as the distance between the centroid point $\left(x_{0}, y_{0}\right)$ and the origin

$$
R(A)=\sqrt{\left(x_{0}\right)^{2}+\left(y_{0}\right)^{2}}
$$

where

$$
x_{0}=\frac{\int_{\operatorname{Supp} A} x \mu_{A}(x) d x}{\int_{\operatorname{Supp} A} \mu_{A}(x) d x}, y_{0}=\frac{\int_{0}^{1} y g_{A}^{L} d y+\int_{0}^{1} y g_{A}^{R} d y}{\int_{0}^{1} g_{A}^{L} d y+\int_{0}^{1} g_{A}^{R} d y}
$$


and $\operatorname{Supp} A$ is the support of $A$.

Fuzzy numbers $A, B$ are then ranked by their ranking function values $R(A)$ and $R(B)$.

\section{CONCLUSIONS}

In this paper we studied the well-known transportation problem and presented several modifications which are important in various application areas.

The assignment problem can also be derived from the linear transportation problem, but it cannot be solved for large instances using linear programming methods and heuristics must be used. We presented a g enetic algorithm (GA) approach and GA parameter settings.

Finally, we studied the crop problem, important in agriculture engineering, and generalised it for case of uncertain crop yields. Instead of traditional interval or PERT approach we propose a fuzzy algebra based on fuzzy numbers and their transformation to scalar values.

In the future, we foresee further tests with other stochastic heuristics, including more applications with special constraints in studied problems.

\section{References}

[1] M. B. Stroh, A Practical Guide to Transportation and Logistics. Dumont: Logistics Network, 2006.

[2] E. Cela, The Quadratic Assignment Problem: Theory and Algorithms. Berlin: Springer, 2010.
[3] Michalewicz, Z:: Genetic Algorithms + Data Structures $=$ Evolution Programs. Springer-Verlag, Berlin, 1996.

[4] M. Šeda, "Mathematical Models of Flow Shop and Job Shop Scheduling Problems." International Journal of Applied Mathematics and Computer Science, nol. 4, no. 4, pp. 241-246, 2007.

[5] M. Šeda, "Heuristic Set-Covering-Based Postprocessing for Improving the Quine-McCluskey Method.” International Journal of Computational Intelligence, vol. 4, no. 2, pp. 139-143, 2007.

[6] D. E. Goldberg, The Design of Innovation (Genetic Algorithms and Evolutionary Computation). Dordrecht: Kluwer Academic Publishers, 2002.

[7] Z. Michalewicz and D.B. Fogel, How to Solve It: Modern Heuristics. Berlin:,Springer-Verlag, 2002.

[8] T. Yamada, C. R. Reeves, "Permutation Flowshop Scheduling by Genetic Local Search,". Int. Conf. Genetic Algorithms in Engineering Systems: Innovations and Applications GALESIA 97. Glasgow, pp. 232-238, 1997

[9] G. Gutin, and A. P. Punnen (eds.). The Traveling Salesman Problem and Its Variations. Dordrecht: Kluwer Academic Publishers, 2002.

[10] N. H. Mitchell, Mathematical Applications in Agriculture, 2nd ed. London: Cengage Learning, 2011.

[11] J Thornley, and J. France, Mathematical Models in Agriculture, 2nd ed., Wallingford, Oxfordshire: CABI, 2006.

[12] A. Bechar, and G. Vitner, "Work Planning in Packing Houses of Flowers Mixed Farms to Increase the yield," Agricultural Engineering International: CIGR Journal, vol. 13, no. 3, 2011, 10 pp., manuscript no. 1901.

[13] V. Novák, Fuzzy Sets and their Applications. Bristol: Adam Hilger, 1989.

[14] C.-H Cheng, "A New Approach for Ranking Fuzzy Numbers by Distance Method," Fuzzy Sets and Systems, vol. 95, pp. 307-317, 1998.

\section{Creative Commons Attribution License 4.0 (Attribution 4.0 International, CC BY 4.0)}

This article is published under the terms of the Creative Commons Attribution License 4.0

https://creativecommons.org/licenses/by/4.0/deed.en_US 